\title{
TRANSITIVITY IN POINT-FREE TOPOLOGY
}

\author{
MOJGAN GOLZY
}

(Received 3 November 2008)

\begin{abstract}
The main purpose of this paper is to develop a point-free notion of topological transitivity. First, we define transitive frame maps and transitive completely prime filters in Frm, the category of frames and frame maps. Then we discuss the relationship between these notions in Frm and the notions of topological transitive and transitive points in Top. Finally, we investigate the relationship between transitive frame maps and the existence of transitive completely prime filters.
\end{abstract}

2000 Mathematics subject classification: primary 06D22; secondary 54H20, 18B30, 18 B35.

Keywords and phrases: point-free topology, frame, transitive frame map, topological transitivity.

\section{Introduction}

Point-free topology deals with certain complete lattices, called frames, which may be viewed as abstractly defined lattices of open sets. It turns out that a remarkable number of topological facts derive from results in this point-free setting while the proofs of the latter are often more suggestive and transparent than those of their classical counterparts [2]. The present paper provides a point-free aspect of topological transitivity.

\section{Background}

In this section we recall some notions and facts which will be used later on. For more details concerning frames we refer to Johnstone [8] or Vickers [12].

2.1. A frame is a complete lattice $L$ in which $a \wedge \bigvee S=\bigvee\{a \wedge t \mid t \in S\}$ for all $a \in L$ and $S \subseteq L$, and a frame homomorphism (simply frame map) is a map $h: L \rightarrow M$ between frames which preserves finitary meets including the top element $e$ and arbitrary joins including the bottom element 0 . The resulting category is denoted by Frm.

A principal example of these notions is the frame $O X$ of open sets of a topological space $X$, and the frame map $f^{-1}[]: O Y \rightarrow O X$, determined by any continuous map

(C) 2009 Australian Mathematical Publishing Association Inc. 0004-9727/2009 \$16.00 
$f: X \rightarrow Y$ between topological spaces. The resulting correspondence constitutes a contravariant functor $O:$ Top $\rightarrow$ Frm.

On the other hand there is a contravariant functor $\Sigma:$ Frm $\rightarrow$ Top such that, for any frame $L, \Sigma L$ is the space of all completely prime filters of $L$, with open sets $\Sigma_{a}=\{F \in \Sigma L \mid a \in F\}, a \in L$. We recall that, a filter $F$ of $L$ is called completely prime filter if, for every $S \subseteq L, \bigvee S \in F$ if and only if $S \cap F \neq \emptyset$. For any frame map $h: L \rightarrow M$, the map $\Sigma h: \Sigma M \rightarrow \Sigma L$ takes $F \in \Sigma M$ to $h^{-1}(F) \in \Sigma L$. Moreover, the contravariant functors $O$ and $\Sigma$ are adjoint on the right, with the adjunction maps $\eta_{L}: L \rightarrow O \Sigma L$ given by $\eta_{L}(a)=\Sigma_{a}$, and $\epsilon_{X}: X \rightarrow \Sigma O X$ given by $\epsilon_{X}(x)=O(x)$ where $O(x)=\{U \in O X \mid x \in U\}$. The frame $L$ is called spatial whenever $\eta_{L}$ is an isomorphism.

2.2. A subset $A$ of a frame $L$ is called a cover of $L$ if $\bigvee A=e$. The set of all covers of $\mathrm{L}$ is denoted by $\operatorname{Cov} L$. The subset $\mathcal{U}$ of $\operatorname{Cov} L$ is called admissible if $a=\bigvee\{x \in L \mid$ $\left.x \triangleleft_{\mathcal{U}} a\right\}$ for each $a \in L$, where $a \triangleleft \mathcal{U} b$ if, for some $C \in \mathcal{U}, \bigvee\{s \in C \mid s \wedge a \neq 0\} \leq b$.

A basis of a frame $L$ is a subset $B \subseteq L$ such that for each $x \in L$,

$$
x=\bigvee\{b \in B \mid b \leq x\} .
$$

A nonzero element $a$ in $L$ is called dense if $a^{*}=0$, where

$$
x^{*}=\bigvee\{z \in L \mid x \wedge z=0\} .
$$

A frame map $h: L \rightarrow M$ is dense if $h(a)=0$ implies $a=0$.

A metric diameter on a frame $L$ is a monotone zero-preserving map $d: L \rightarrow \bar{R}_{+}$, the extended nonnegative reals, such that:

(1) $\quad d(a \vee b) \leq d(a)+d(b)$ if $a \wedge b \neq 0$;

(2) for each $\epsilon>0, D_{\epsilon}=\{a \in L \mid d(a)<\epsilon\}$ is a cover;

(3) for all $a \in L$ and $\epsilon>0, d(a)=\sup \left\{d(x \vee y) \mid x, y \leq a, x, y \in D_{\epsilon}\right\}$;

(4) the set of these covers is admissible.

A metric frame is a frame equipped with a specified metric diameter. For any topological space $X$, the frame $O X$ has the metric diameter $d$ on $L$ corresponding exactly to the usual metrization $\rho$ of $X$ by the relations

$$
d(U)=\sup \{\rho(x, y) \mid x, y \in U\}, \quad \rho(x, y)=\inf \{d(U) \mid x, y \in U\} .
$$

Hence, the metric diameters are exactly the point-free counterparts of metrics in classical topology [4].

A frame $L$ is compact if $\bigvee S=e$ implies $\bigvee T=e$ for some finite $T \subseteq S$. A frame $L$ is regular if $a=\bigvee\{x \in L \mid x \prec a\}$ for each $a \in L$ where $x \prec a$ (x rather below $a$ ) means $a \vee x^{*}=e$. For a topological space $X$, the frame $O X$ is compact (respectively regular) if and only if $X$ is compact (respectively regular) [6].

A frame $L$ is called continuous (or locally compact) whenever $a=\bigvee\{x \mid x \ll a\}$ for each $a \in L$ where $x \ll a(x$ way below $a)$ means, for any $S \subseteq L, a \leq \bigvee S$ implies 
$x \leq \bigvee F$, for some finite set $F \subseteq S$. For a topological space $X$, the frame $O X$ is continuous if $X$ is locally compact and if $X$ is a regular locally compact space then $O X$ is a regular continuous frame [6].

\section{Transitive completely prime filters}

In this section we define the notion of transitive completely prime filters in Frm and then we study the relationship between these filters and transitive points in Top.

We recall that for a continuous map $f: X \rightarrow X$ with $X$ a topological space, any point with dense orbit under $f$ is called a transitive point of $f$, where the orbit of $x$ under $f$ is given by $O_{f}(x)=\left\{f^{n}(x) \mid n=0,1, \ldots,\right\}$. The set of transitive points of $f$ is denoted by $\operatorname{tr}(f)[9,11]$.

Definition 3.1. Let $h: L \rightarrow L$ be a frame map. We say a completely prime filter $F$ of $L$ is transitive under $h$ if, for each nonzero element $a$ in $L$, there is a nonnegative integer $n$ such that $h^{n}(a) \in F$. We denote by $T(h)$, the set of all transitive completely prime filters under $h$.

Proposition 3.2. Let $X$ be a topological space and $f: X \rightarrow X$ a continuous map. Then $x \in \operatorname{tr}(f)$ if and only if $O(x) \in T\left(f^{-1}[]\right)$, where $O(x)=\{U \in O X \mid x \in U\}$.

Proof. Given $x \in \operatorname{tr}(f)$ it is easy to see that $O(x)$ is a completely prime filter. Since $\left\{x, f(x), f^{2}(x), \ldots\right\}$ is dense in $X$, for each nonempty $V \in O X$, there is a nonnegative integer $n$ such that $f^{n}(x) \in V$. Therefore $f^{-n}(V) \in O(x)$. Hence $O(x)$ is transitive under $f^{-1}$ [ ]. Conversely, $O(x)$ being transitive implies that for each nonempty $V \in O X$ there is a nonnegative integer $n$ such that $f^{-n}(V) \in O(x)$ which implies $f^{n}(x) \in V$. Thus $x$ is a transitive point of $f$.

Proposition 3.3. Let $h: L \rightarrow L$ be a frame map. Then $T(h) \subseteq \operatorname{tr}(\Sigma h)$. Moreover, $T(h)=\operatorname{tr}(\Sigma h)$ if $\eta_{L}$ is dense.

Proof. Given $P \in T(h)$ and a nonempty open set $\Sigma_{a}$ of $\Sigma L, \Sigma_{a} \neq \emptyset$ implies $a \neq 0$. So there is an integer $n \geq 0$ such that $h^{n}(a) \in P$ or equivalently $a \in$ $h^{-n}(P)=(\Sigma h)^{n}(P)$. Thus $\left\{(\Sigma h)^{n}(P) \mid n \geq 0\right\} \cap \Sigma_{a} \neq \emptyset$ which implies $P \in \operatorname{tr}(\Sigma h)$. Conversely, let $\eta_{L}$ be dense and $P \in \operatorname{tr}(\Sigma h)$. Given $a \neq 0$. Since $\eta_{L}(a)=\Sigma_{a} \neq \emptyset$ there is a nonnegative integer $n$ such that $a \in h^{-n}(P)=(\Sigma h)^{-n}(P)$. Therefore, $h^{n}(a) \in P$, and so $P \in T(h)$.

LEMMA 3.4. Let $h: L \rightarrow L$ be a frame map. If $T(h) \neq \emptyset$, then $\eta_{L}$ is dense.

Proof. Given $P \in T(h)$ and $a \neq 0$ in $L$ there is $n \geq 0$ such that $h^{n}(a) \in P$ and so $h^{-n}(P) \in \Sigma_{a}=\eta_{L}(a)$. Hence, $\eta_{L}$ is dense.

COROllary 3.5. For a frame map $h: L \rightarrow L$, if $T(h) \neq \emptyset$ then $T(h)=\operatorname{tr}(\Sigma h)$. 
Proposition 3.6. Let $B$ be a basis for the frame $L$, and $h: L \rightarrow L$ a frame map. Then a completely prime filter $F$ is transitive under $h$ if and only if for each nonzero element $b$ in $B$ there is a nonnegative integer $n$ such that $h^{n}(b) \in F$.

Proof. Given $0 \neq x \in L$ there is $0 \neq b \in B$ such that $b \leq x$, since $x=\bigvee\{b \in B \mid$ $b \leq x\}$. Take $n \geq 0$ such that $h^{n}(b) \in F$ and so $b \in h^{-n}(F)$. Since $h^{-1}$ preserves completely prime filters $h^{-n}(F)$ is also a completely prime filter. Thus $x \in h^{-n}(F)$ or equivalently $h^{n}(x) \in F$. Therefore, $F$ is transitive. The converse is trivial.

\section{Transitive frame maps}

In this section we introduce the notion of transitive frame maps and then we investigate the relationship between these frame maps and topologically transitive maps.

We recall that, a continuous map $f: X \rightarrow X$, with $X$ a topological space, is topologically transitive if, for every pair of nonempty open sets $U$ and $V$ in $X$, there is a positive integer $n$ such that $f^{n}(U) \cap V \neq \emptyset[5]$.

Definition 4.1. Let $L$ be a frame and $h: L \rightarrow L$ a frame map. We say $h$ is transitive if, for every pair of nonzero elements $a$ and $b$ of $L$, there is a positive integer $n$ such that $\Sigma_{a \wedge h^{n}(b)} \neq 0$. That is, there is a completely prime filter $P$ such that $a \wedge h^{n}(b) \in P$.

Proposition 4.2. Let $f: X \rightarrow X$ be a continuous map, with $X$ a topological space. Then $f$ is topologically transitive if and only if $f^{-1}[]: O X \rightarrow O X$ is a transitive frame map.

\section{PROOF.}

$\Rightarrow$ Given nonempty open sets $U$ and $V$ in $X$ there is a positive integer $n$ such that $f^{n}(U) \cap V \neq \emptyset$. We claim $\sum_{U \cap f^{-n}(V)} \neq \emptyset$. Given $x \in f^{n}(U) \cap V$ there is $y \in U$ such that $f^{n}(y)=x$. Take $O(y)=\{W \in O X \mid y \in W\}$. Note that, $O(y)$ is a completely prime filter which contains $U$ and $f^{-n}(V)$. Hence $O(y) \in \sum_{U \cap f^{-n}(V)}$. Therefore, $f^{-1}[$ ] is a transitive frame map.

$(\Leftarrow)$ Given nonempty open sets $U$ and $V$ in $X$ there is a positive integer $n$ such that $\sum_{U \cap f^{-n}(V)} \neq \emptyset$. Thus $U \cap f^{-n}(V) \neq \emptyset$ and so $f^{n}(U) \cap V \neq \emptyset$. Therefore $f$ is topologically transitive.

PROposition 4.3. If $h: L \rightarrow L$ is a transitive frame map then $\Sigma h: \Sigma L \rightarrow \Sigma L$ is topologically transitive.

Proof. Given $U, V \neq \varnothing$ in $O(\Sigma L)$ there are $a, b \neq 0$ in $L$ such that $U=\Sigma_{a}$ and $V=\Sigma_{b}$. Since $h$ is transitive, there is a positive integer $n$ and a completely prime filter $P$ such that $h^{n}(a) \wedge b \in P$. Thus $h^{-n}(P) \in \Sigma_{a}$ and

$$
h^{-n}(P) \in\left\{h^{-n}(Q) \mid b \in Q \text { and } Q \in \Sigma L\right\}=(\Sigma h)^{n}\left(\Sigma_{b}\right) .
$$

Therefore, $h^{-n}(P) \in \Sigma_{a} \cap(\Sigma h)^{n}\left(\Sigma_{b}\right) \neq \emptyset$. Hence $\Sigma h$ is topologically transitive. 
Proposition 4.4. Let $h: L \rightarrow L$ be a transitive frame map. Then the following are true.

(i) The frame map $h$ is dense.

(ii) The adjunction map $\eta_{L}$ is dense.

(iii) For each $a \neq 0, \bigvee\left\{h^{n}(a) \mid n=1,2, \ldots\right\}$ is dense.

(iv) If $h(a)<a$, then $a$ is dense.

PROOF.

(i) Given $a \neq 0$ there is $n \geq 1$ such that $\sum_{e \wedge h^{n}(a)} \neq \emptyset$ which implies $h(a) \neq 0$.

(ii) Given $a \neq 0$ there is $n \geq 1$ such that $\sum_{a \wedge h^{n}(e)} \neq \emptyset$ which implies $\eta_{L}=\Sigma_{a} \neq \emptyset$.

(iii) Let $a \neq 0$. For each $b \neq 0$, there is $n \geq 1$ such that $\sum_{b \wedge h^{n}(a)} \neq \emptyset$ and so $b \wedge h^{n}(a) \neq 0$. Thus $b \wedge \bigvee\left\{h^{n}(a) \mid n=1,2, \ldots\right\} \neq 0$. Therefore $\bigvee\left\{h^{n}(a) \mid n=\right.$ $1,2, \ldots\}$ is dense.

(iv) Let $h(a)<a$. Since $a \neq 0$, for each $b \neq 0$, there is $n \geq 1$ such that $\sum_{b \wedge h^{n}(a)} \neq \emptyset$ and so $b \wedge h^{n}(a) \neq 0$. Thus $b \wedge a \neq 0$. Therefore, $a$ is dense.

Proposition 4.5. Let $h: L \rightarrow L$ be a frame map. The following are equivalent.

(i) The frame map $h$ is transitive.

(ii) The adjunction map $\eta_{L}$ is dense and, for each $a \neq 0, \bigvee\left\{h^{n}(a) \mid n=1,2, \ldots\right\}$ is dense.

(iii) The adjunction map $\eta_{L}$ is dense and $\Sigma_{h}: \Sigma L \rightarrow \Sigma L$ is topologically transitive.

PROOF. By the previous proposition, (i) implies (ii) and (iii); also (ii) implies (i). Suppose that $a \neq 0$ and $b \neq 0$. Since $\bigvee\left\{h^{n}(b) \mid n=1,2, \ldots\right\}$ is dense, $a \wedge$ $\bigvee\left\{h^{n}(b) \mid n=1,2, \ldots\right\} \neq 0$. Thus, there is $n \geq 1$ such that $a \wedge h^{n}(b) \neq 0$. Therefore, $\sum_{a \wedge h^{n}(b)} \neq \emptyset$, since $\eta_{L}$ is dense.

To prove that (iii) implies (i), we proceed as follows. Suppose that $a \neq 0$ and $b \neq 0$. Since $\eta_{L}$ is dense $\Sigma_{a} \neq \emptyset$ and $\Sigma_{b} \neq \emptyset$. Thus there is $n \geq 1$ such that $\Sigma_{b} \cap(\Sigma h)^{n}\left(\Sigma_{a}\right) \neq \emptyset$. Take $P \in \Sigma_{b} \cap(\Sigma h)^{n}\left(\Sigma_{a}\right)$. Then $b \in P$ and $P \in\left\{h^{-n}(Q) \mid\right.$ $a \in Q$ and $Q \in \Sigma L\}$. However, there is a completely prime filter $Q$ such that $a \in Q$ and $P=h^{-n}(Q)$. Hence, $a \wedge h^{n}(b) \in Q$ which implies $\Sigma_{a \wedge h^{n}(b)} \neq \emptyset$. Therefore, $h$ is transitive.

\section{Relationships}

In this section we investigate the relationship between transitive frame maps and the existence of transitive completely prime filters.

Note that, some authors give different definitions for topological transitivity for compact metric spaces: a continuous map $f: X \rightarrow X$ is topologically transitive if there is a point $x \in X$ whose orbit is dense in $X$; that is, $\operatorname{tr}(f) \neq \varnothing[1]$. This definition is not equivalent to the definition that we adopt in this paper. Under some additional assumptions on the phase space or on the map, the two definitions are equivalent [5, 10]. From a point-free perspective also, the existence of transitive completely prime filters does not coincide with the transitivity of the frame map. 
EXAMPLE 5.1. Consider the frame $L=\left(\left\{0, a, a^{\prime}, e\right\}, \leq\right)$ and the frame map $h: L \rightarrow$ $L$ given by $h(0)=h(a)=0, h(e)=h\left(a^{\prime}\right)=e$. Then $\{a, e\} \in T(h)$ implies $T(h) \neq \emptyset$ but $h$ is not transitive, since it is not dense. In this example $L$ is a compact regular frame.

Proposition 5.2. Let $h: L \rightarrow L$ be a dense frame map. If $T(h) \neq \emptyset$ then $h$ is transitive.

Proof. Given nonzero elements $a, b$ in $\mathrm{L}$ and $P \in T(h)$ there is $n \geq 0$ such that $h^{n}(a) \in P$. Since $h$ is dense $h^{n+1}(b) \neq 0$ and so there is $m \geq 0$ such that $h^{m}\left(h^{n+1}(b)\right) \in P$. Take $t=m+1$. Then $a \in h^{-n}(P)$ and $h^{t}(b) \in h^{-n}(P)$. Therefore $h^{-n}(P) \in \Sigma_{a \wedge h^{t}(b)}$, for some $t \geq 1$. Hence $h$ is transitive.

We will use the following facts in the next proposition.

(1) For any completely prime filter $F$ of a frame $L$, there is a prime element $p$ such that $F=L-\downarrow p$, where $p$ is prime, if $p \neq e$ and $p=x \wedge y$ implies $p=x$ or $p=y$ and $\downarrow p=\{x \mid x \leq p\}[7]$.

(2) For regular frames the set of prime elements coincides with the set of coatoms; maximal elements differ from the top element [6].

PROPOSITION 5.3. Let $L$ be a regular frame with no atom and $h: L \rightarrow L$ a frame map. Then $T(h) \neq \emptyset$ implies $h$ is transitive.

Proof. By Proposition 5.2, it is enough to show that $h$ is dense. Let $a \neq 0$ with $h(a)=0$. Take $F \in T(h)$. There is a prime element $p \in L$ such that $F=L-\downarrow p$. Note that, $h(a \wedge p) \leq h(a)=0$ and $F \in T(h)$ imply $a \in F$ and $a \wedge p=0$. If $a \wedge c=$ 0 then $c \leq p$. Thus $p=a^{*}$. Now, let $0<b<a$. Then $b \in F$. Since $L$ is regular, $p$ is a coatom, and so $e=b \vee p=b \vee a^{*}$. Thus $a \prec b$ which implies $a \leq b$. This shows that $a$ is an atom. This contradicts the hypothesis and so we conclude that $h$ is dense.

We will use the following facts in the next proposition.

(1) In a compact regular frame, $a \ll b$ if and only if $a \prec b$ and so a compact regular frame is continuous.

(2) A compact regular frame is metrizable if and only if it has a countable basis [3].

(3) The Baire category theorem for continuous lattices is as follows. Let $L$ be any continuous semilattice with a smallest element 0 and $D$ a countable collection of dense elements. Then for any nonzero element $u$ there is a prime element $p$ such that $u \wedge v \not \leq p$, for each $v \in D$ [7].

Proposition 5.4. Let $L$ be a compact metric frame and $h: L \rightarrow L$ a transitive frame map. Then $T(h) \neq \emptyset$.

PROOF. Since $L$ is a compact metric frame it is continuous and has a countable basis, say $B$. Take $C=\left\{\bigvee_{n=1}^{\infty} h^{n}(b) \mid b \in B\right\}$. By Proposition 4.4, $C$ is a countable set of dense elements of $L$ and so by the Baire category theorem for continuous lattices, there is a prime element $p$ such that $e \wedge v \in L-\downarrow p$, for each $v \in C$. Thus $F=L-\downarrow p$ is 
a completely prime filter in which for each $b \in B$ there is a positive integer $n$ such that $h^{n}(b) \in F$. Hence $F \in T(h)$.

REMARK 5.5. The above proposition also can be proved by the following facts: compact metric frames are exactly the point-free counterparts of compact metric in classical topology; the topological transitivity of a continuous map in compact metric spaces implies the existence of transitive points under the map.

\section{Acknowledgement}

I would like to thank the referee for their valuable suggestions.

\section{References}

[1] E. Akin, The General Topology of Dynamical Systems, Graduate Studies in Mathematics, 1 (American Mathematical Society, Providence, RI, 1993).

[2] B. Banaschewski, Uniform Completion in Point-free Topology, Lecture Notes in Mathematics (University of Cape Town, Cape Town, 1996), pp. 1-26.

[3] B. Banaschewski and A. Pultr, 'A new look at pointfree metrization theorems', Comment. Math. Univ. Carolin. 39(1) (1998), 167-176.

[4] _ ' 'A Stone duality for metric spaces', in: Category Theory, Montreal Quebec, 1991, Canadian Mathematical Society Conference Proceedings, 13 (American Mathematical Society, Providence, RI, 1992), pp. 33-42.

[5] L. S. Block and W. A. Coppel, Dynamics in One Dimension, Lecture Notes in Mathematics, 1513 (Springer, Berlin, 1992).

[6] M. M. Ebrahimi and M. Mahmoudi, 'Frames (I)', Technical Report, Shahid Beheshti University, 1995.

[7] G. Gierz, K. H. Hofmann, K. Keimel, J. D. Lawson, M. W. Mislove and D. S. Scott, Continuous Lattices and Domains (Cambridge University Press, Cambridge, 2003).

[8] P. T. Johnstone, Stone Spaces (Cambridge University Press, Cambridge, 1982).

[9] S. Kolyada and L. Snoha, 'Some aspects of topological transitivity-a survey', in: Iteration Theory, ECIT'94, Opava, Grazer Mathematische Beridite, 334 (Karl-Franzens-Universität, Graz, 1997), pp. 3-35.

[10] K. Petersen, Ergodic Theory (Cambrige University Press, Cambridge, 1983).

[11] D. Pokluda, 'On the structure of sets of transitive points for continuous maps of the intervals', Real Anal. Exchange 25 (1999/2000), 45-48.

[12] S. Vickers, Topology via Logic, Cambridge Tracts in Theoretical Computer Science, 5 (Cambridge University Press, Cambridge, 1985).

MOJGAN GOLZY, Department of Mathematics, Buffalo State College, 1300 Elmwood Avenue, 317 Bishop Hall, NY 14222, USA

e-mail: golzym@buffalostate.edu 